\section{'Liuyuezao': A New Very Early-ripening Pummelo Cultivar}

Tengfei Pan and Peibin Huang

College of Horticulture, Fujian Agriculture and Forestry University, Fuzhou, Fujian 350002, China

Jianwen Ye

Pinghe County Farm Bureau, Pinghe, Fujian 363700, China

Dongming Pan

College of Horticulture, Fujian Agriculture and Forestry University, Fuzhou, Fujian 350002, China

\section{Zhijun Fu}

College of Horticulture, Fujian Agriculture and Forestry University, Fuzhou, Fujian 350002, China; and FAFU-UCR Joint Center for Horticultural Biology and Metabolomics, Haixia Institute of Science and Technology, Fujian Agriculture and Forestry University, Fuzhou, Fujian 350002, China

\section{Heli Pan, Zhixiong Guo, and Wenqin She}

College of Horticulture, Fujian Agriculture and Forestry University, Fuzhou, Fujian 350002, China

\section{Yuan Yu}

College of Horticulture, Fujian Agriculture and Forestry University, Fuzhou, Fujian 350002, China; and FAFU-UCR Joint Center for Horticultural Biology and Metabolomics, Haixia Institute of Science and Technology, Fujian Agriculture and Forestry University, Fuzhou, Fujian 350002, China

Additional index words. Citrus maxima, early season, fruit breeding season cultivar developed from a spontaneous bud sport on a 'Guanxi' pummelo tree. Trees of 'Liuyuezao' are moderately large and vigorous in their growth habit. The fruit mature from late July to early August in Fujian, 40 to $60 \mathrm{~d}$ ahead of 'Guanxi', 'Hongrou', and 'Sanhong' pummelo. The average yield is 68.23 to $70.88 \mathrm{~kg} / \mathrm{plant}$; the average weight is 1213.17 to $1228.95 \mathrm{~g} /$ fruit. The fruit has a light-yellow, smooth rind. The flesh is pale yellow, tender, and juicy, with a sweet and mildly acidic flavor without bitterness. Fruit quality characteristics were measured for 'Liuyuezao', including the soluble solids content $(10.86$ ${ }^{\circ}$ Brix), total organic acid content $(10.89 \mathrm{mg} / \mathrm{g})$, and vitamin $\mathrm{C}$ content (45.87 $\mathrm{mg} / 100 \mathrm{~g})$. 'Liuyuezao' is suited for planting in Fujian,

Received for publication 7 Aug. 2021. Accepted for publication 19 Sept. 2021.

This study was supported financially by the National Natural Science Foundation of China (grant no. 31972369) to Y.Y. and the startup fund from the Fujian Agriculture and Forestry University Horticulture Science Plateau Discipline Construction Project (grant no. 712018011).

T.P. and P.H. are co-first authors.

Y.Y. is the corresponding author. E-mail: yyu@ fafu.edu.cn or ymmzyz@hotmail.com.

This is an open access article distributed under the org/licenses/by-nc-nd/4.0/).
'Liuyuezao' pummelo is a new very early-

Published online 9 November 2021. CC BY-NC-ND license (https://creativecommons.
Liuyuezao' is suggested for planting in Fujian, Guangdong, Guangxi, and Hainan, and at low altitudes in South Yunnan in China.

\section{Origins}

'Liuyuezao' originated from a spontaneous bud sport on a 'Guanxi' pummelo tree in 2000 in a pummelo orchard in Xiaoxi, Pinghe, Fujian. Budwood was taken from the bud sport branch and was grafted on sour pummelo ( $C$. maxima) rootstock in a commercial pummelo orchard in Pinghe, Fujian, in 2009. 'Liuyuezao' was planted in several commercial pummelo orchards in Pinghe, Shanghang, Zhangpu, and Yongchun of Fujian, and was observed for several generations and found to be stable.

\section{Plant Characteristics}

Trees of 'Liuyuezao' are moderately large, vigorous, and spreading in their growth habit, with semicircular crowns. Young shoots are green, triangular, and usually with thorns, but then become dark green and round when they mature. Bark and branch bark color is taupe. 'Liuyuezao' flowers occur singly or in clusters, and are complete flowers with white petals; thick, white filaments; yellow anthers; and abundant pollen. The style is purple-red, and the anther length is shorter than the stigma.

From 2018 to 2019, plant yield, and bloom and ripening dates of 'Liuyuezao', 'Guanxi', 'Hongrou', and 'Sanhong' were observed and measured (Table 1). The main flushes of 'Liuyuezao' arise in spring and fall, with the spring flush being mainly fruit-bearing. Spring and fall buds begin to sprout in late March and early August, respectively. 'Liuyuezao' trees usually flower in March, with a high fruit setting rate from self-pollination. 'Liuyuezao' starts fruiting from early April to early August, and the fruit ripen from late July to early August in Fujian, about 40 to $60 \mathrm{~d}$ earlier than 'Guanxi', 'Hongrou', and 'Sanhong' (Fig. 1). Our comparison showed that the yields of 'Liuyuezao' (68.23-70.88 kg/plant), 'Guanxi' (68.73-70.22 $\mathrm{kg}$ /plant), 'Hongrou' (69.48-70.08 kg/plant), and 'Sanhong' (74.95-75.65 kg/plant) were not significantly different from each other within each harvest (Table 1). Stable and excellent performance of 'Liuyuezao' was observed, demonstrating moderately vigorous plant growth, strong branching capacity, high fruit setting rate, extremely early ripening, heavy crop, and fine eating quality.

\begin{tabular}{lllcc}
\hline & & \multicolumn{2}{c}{ Yield (kg/plant) } \\
\cline { 3 - 5 } Cultivar $^{z}$ & Flowering time & Fruit ripening time & 2018 & \multicolumn{1}{c}{2019} \\
\hline Liuyuezao & Early to mid March & Early August & $68.23 \pm 10.46 \mathrm{a}$ & $70.88 \pm 9.75 \mathrm{a}$ \\
Guanxi & Mid to late March & Late September & $70.22 \pm 10.76 \mathrm{a}$ & $68.73 \pm 10.88 \mathrm{a}$ \\
Hongrou & Mid to late March & Late September & $70.08 \pm 11.94 \mathrm{a}$ & $69.48 \pm 8.03 \mathrm{a}$ \\
Sanhong & Mid to late March & Late September & $75.65 \pm 5.76 \mathrm{a}$ & $74.95 \pm 4.97 \mathrm{a}$ \\
\hline
\end{tabular}

The data of yield are the mean \pm SD of six trees per cultivar: same letters in the same columns mean and the flesh is soft, juicy, and flavorful -
Table 1. Flowering and fruit ripening time and yield of 'Liuyuezao', 'Guanxi', 'Hongrou', and 'Sanhong' pummelo in Pinghe, Fujian, 2018-19.

${ }^{\mathrm{z}}$ All the trees were grafted on sour pummelo rootstock in 2009. not significantly different according to Tukey's honestly significant difference test at $P<0.05$.

Guangdong, Guangxi, and Hainan, and at low titudes in South Yunnan, China.

an important local economy in Fujian Province, China, where the total production in $90 \%$ of the planted pummelo trees in fruit of all the three cultivars 2016; Zhu et al., 2020). 'Liuyuezao' pummelo 20201003385) is a new very season cultivar developed by the Fujian Agriculture and Forestry University. The fruit (a) 


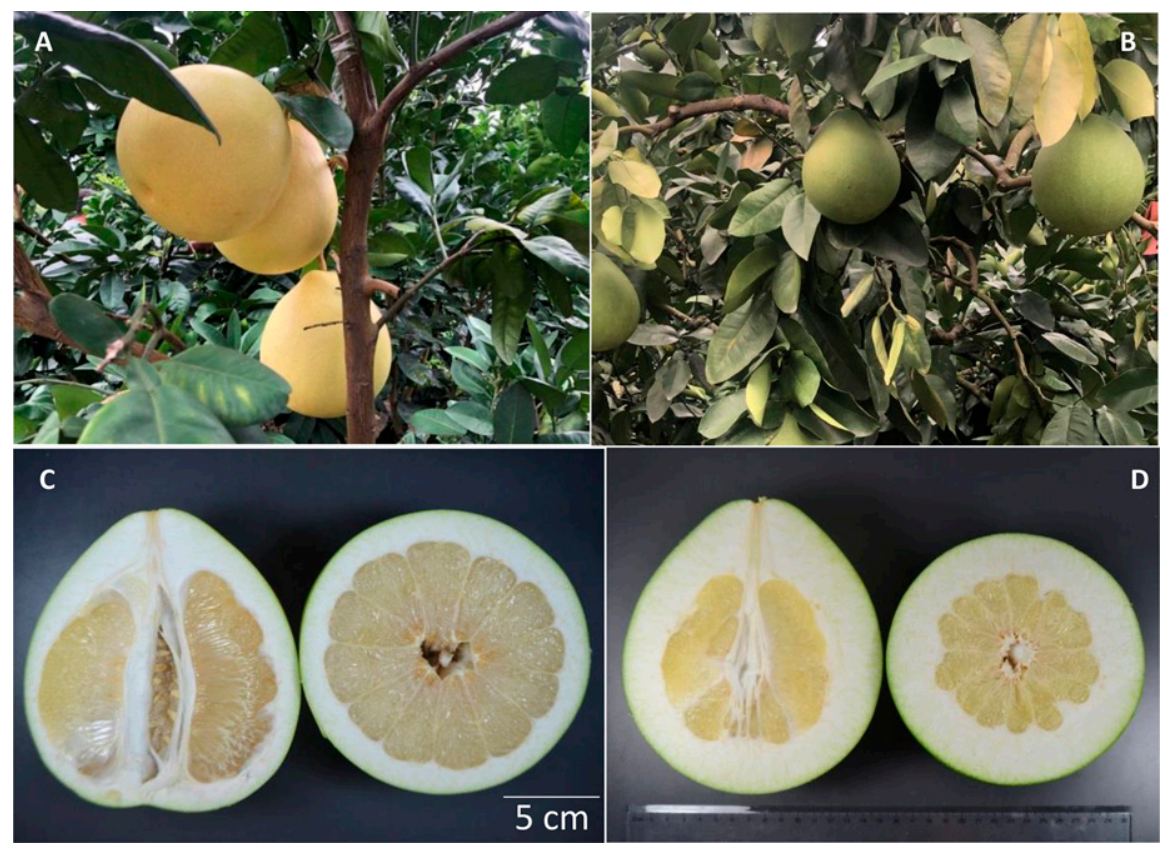

Fig. 1. Tree-hung and cut fruit traits of 'Liuyuezao' and 'Guanxi' pummelo in Pinghe, Fujian, on 6 Aug. 2018. (A) Typical ripe 'Liuyuezao' fruit on a 9-year-old tree on sour pummelo rootstock. (B) Green 'Guanxi' fruit on a 9-year-old tree on sour pummelo rootstock. (C) Freshly harvested mature 'Liuyuezao' fruit cut longitudinally and equatorially, respectively. (D) Freshly harvested green 'Guanxi' fruit cut longitudinally and equatorially, respectively.

Table 2. Fruit attributes of 'Liuyuezao', 'Guanxi', 'Hongrou', and 'Sanhong' pummelo in Pinghe, Fujian, 2018-19.

\begin{tabular}{lcccccc}
\hline Cultivar $^{\mathrm{z}}$ & Fruit ht $(\mathrm{cm})$ & Fruit diam $(\mathrm{cm})$ & Fruit wt $(\mathrm{g})$ & Soluble solids $\left({ }^{\circ}\right.$ Brix $)$ & Total organic acids $(\mathrm{mg} / \mathrm{g})$ & Vitamin C $(\mathrm{mg} / 100 \mathrm{~g})$ \\
\hline \multicolumn{7}{c}{} \\
\hline Liuyuezao & $15.08 \pm 0.96 \mathrm{~b}$ & $13.91 \pm 0.98 \mathrm{~b}$ & $1213.17 \pm 135.9 \mathrm{~b}$ & $10.68 \pm 1.73 \mathrm{a}$ & $11.41 \pm 0.65 \mathrm{a}$ & $43.03 \pm 3.36 \mathrm{a}$ \\
Guanxi & $14.23 \pm 1.08 \mathrm{bc}$ & $14.78 \pm 0.71 \mathrm{~b}$ & $1258.7 \pm 130.67 \mathrm{~b}$ & $10.71 \pm 0.35 \mathrm{a}$ & $8.01 \pm 0.62 \mathrm{bc}$ & $39.57 \pm 3.47 \mathrm{a}$ \\
Hongrou & $13.6 \pm 0.8 \mathrm{c}$ & $13.64 \pm 0.47 \mathrm{~b}$ & $1109.99 \pm 184.26 \mathrm{~b}$ & $13.02 \pm 0.3 \mathrm{a}$ & $8.56 \pm 0.59 \mathrm{~b}$ & $34.79 \pm 6.63 \mathrm{a}$ \\
Sanhong & $16.99 \pm 1.3 \mathrm{a}$ & $16.73 \pm 1.39 \mathrm{a}$ & $1442.07 \pm 113.41 \mathrm{a}$ & $11.74 \pm 0.25 \mathrm{a}$ & $6.96 \pm 0.29 \mathrm{c}$ & $41.3 \pm 5.11 \mathrm{a}$ \\
\hline \multicolumn{7}{c}{2019} \\
\hline Liuyuezao & $15.57 \pm 1.16 \mathrm{a}$ & $14.08 \pm 0.78 \mathrm{a}$ & $1228.95 \pm 166.38 \mathrm{a}$ & $11.03 \pm 0.15 \mathrm{a}$ & $10.37 \pm 0.46 \mathrm{a}$ & $48.71 \pm 4.09 \mathrm{a}$ \\
Guanxi & $13.92 \pm 1.29 \mathrm{~b}$ & $15.14 \pm 1.49 \mathrm{a}$ & $1218.97 \pm 145.97 \mathrm{a}$ & $9.94 \pm 0.08 \mathrm{~b}$ & $8.16 \pm 0.46 \mathrm{~b}$ & $42.08 \pm 6.21 \mathrm{a}$ \\
Hongrou & $15.26 \pm 1.04 \mathrm{ab}$ & $13.98 \pm 1.03 \mathrm{a}$ & $1105.53 \pm 168.2 \mathrm{a}$ & $11.3 \pm 0.27 \mathrm{a}$ & $8.73 \pm 0.76 \mathrm{ab}$ & $41 \pm 6.8 \mathrm{a}$ \\
Sanhong & $15.69 \pm 0.87 \mathrm{a}$ & $14.56 \pm 0.94 \mathrm{a}$ & $1223.41 \pm 121.58 \mathrm{a}$ & $10.85 \pm 0.35 \mathrm{a}$ & $7.32 \pm 0.87 \mathrm{~b}$ & $44.2 \pm 5.14 \mathrm{a}$ \\
\hline
\end{tabular}

${ }^{\mathrm{z}}$ All the trees were grafted on sour pummelo rootstock in 2009.

All values are mean \pm SD of six samples (three fruits each) per cultivar. Different letters in same column and within a harvest season indicate significant differences according to Tukey's honestly significant difference test at $P<0.05$.

\section{Fruit Quality Comparisons}

'Liuyuezao' fruit are pear-shaped with a slightly flattened bottom and have a light-yellow external color (Fig. 1). The rind is moderately thick with a smooth surface that is tightly adherent. They have 12 to 15 segments with moderately thick and tough carpellary membranes that are difficult to separate. The fruit develops a pale-yellow flesh that is tender, juicy, sweet, and mildly acidic without bitterness. Compared with 'Guanxi', 'Liuyuezao' displayed a similar fruit diameter $(14 \mathrm{~cm})$, fruit weight (1221.06 g), and vitamin $\mathrm{C}$ content $(45.87 \mathrm{mg} / 100 \mathrm{~g})$, but a significantly greater organic acid content $(10.89 \mathrm{mg} / \mathrm{g})$. In addition, 'Liuyuezao' fruit exhibited a soluble solids content $\left(10.86^{\circ}\right.$ Brix $)$ similar to the other two mutants 'Hongrou' and 'Sanhong' (Table 2).

\section{Availability}

The cultivar is tested and maintained in the orchard at the College of Horticulture, Fujian Agriculture and Forestry University, Fuzhou, Fujian, China. Requests for the cultivar and prospective licensees should contact Tengfei Pan (e-mail: tfpan@fafu.edu.cn) at the College of Horticulture, Fujian Agriculture and Forestry University, Fuzhou, Fujian 350002, China.

\section{Literature Cited}

Liu, W., Q. Ye, X. Jin, F. Han, X. Huang, S. Cai, and L. Yang. 2016. A spontaneous bud mutant that causes lycopene and beta-carotene accumulation in the juice sacs of the parental Guanxi pummelo fruits [Citrus grandis (L.) Osbeck]. Scientia Hort. 198: 379-384, https://doi.org/10.1016/j.scienta. 2015.09.050.

Zhu, C., Q. Lu, X. Zhou, J. Li, J. Yue, Z. Wang, and S. Pan. 2020. Metabolic variations of organic acids, amino acids, fatty acids and aroma compounds in the pulp of different pummelo varieties. Lebensm. Wiss. Technol. 130:109445, https://doi.org/10.1016/j.lwt.2020.109445. 